Conclusion This classification system can be useful to accurately describe laryngoscopic findings during Ilo assessment. This system is now incorporated into our reporting practices to increase capture of Ilo diagnosis. The inclusion of supraglottic presentation with symptoms supports accurate diagnosis and treatment and further understanding of Ilo.

\section{P23 PATIENT-REPORTED ONSET FACTORS IN INDUCIBLE LARYNGEAL OBSTRUCTION}

${ }^{1} \mathrm{C}$ Slinger, ${ }^{2} \mathrm{R}$ Slinger, ${ }^{1} \mathrm{~A}$ Vyas. 'Lancashire Teaching Hospitals, Preston, UK; ${ }^{2}$ Lancaster University, Lancaster, UK

\subsection{6/thoraxjnl-2017-210983.165}

Introduction and Objectives Inducible Laryngeal Obstruction (Ilo) is poorly understood, in terms of aetiology and onset by patients and clinicians. Patients presenting to our Tertiary Airways service commonly seek an understanding of the causes and triggers of their Ilo episodes. This study aims to develop a taxonomy of categories of patient-reported onset factors for Ilo from a sample of patients with confirmed Ilo on laryngoscopy, referred to our service.

Method Within a nine month period, 103 patients referred to the Airways service $(76 \%$ female, $24 \%$ male; age range $15-86$ years $($ median $=52)$ ) with endoscopically-confirmed Ilo were asked to report historical factors contemporaneous with the initial onset of Ilo symptoms. A retrospective analysis of patient notes was also conducted to identify co-morbidities and additional onset factors. Single or multiple onset factors for each patient were collected, which were coded into initial themes. From these themes, second-order onset categories were developed which incorporated factors reported by all participants.

Results Thirteen initial onset themes were developed. These included psychological factors (25\% of patients), upper respiratory tract infections (23\%), reflux (17\%), chest infections (14\%), medical conditions (12\%) and surgery (10\%). These were then refined into a taxonomy of five categories of onset factors:

- Respiratory Tract Infections and Viruses (40\% of patients)

- Underlying Medical Conditions, e.g., rhinitis with post nasal drip, reflux (34\%)

- Psychological difficulties (25\%)

- Irritants, e.g., medication (23\%)

- Exercise (7\%)

Only one patient had onset factors in multiple categories, indicating that these categories are largely independent of each other. The prevalence of certain onset factors was mediated to some degree by age, e.g., medical conditions were more frequent factors for older participants, whereas exercise was a more common factor in younger participants.

Conclusions This study demonstrates patient-reported onset factors for Ilo can be usefully translated into a detailed taxonomy based on specific conditions/triggers. This understanding may be useful in furthering our understanding, both of common trigger and onset factors that can be communicated to patients, and may help to inform therapeutic interventions aimed at the active self-management of Ilo.

\section{Clinical update in COPD}

\section{P24 MAPPING OF END OF LIFE RECOGNITION AND PALLIATIVE CARE PROVISION IN COPD}

${ }^{1} \mathrm{HM}$ Ward, ${ }^{2} \mathrm{~A}$ Wood, ${ }^{3} \mathrm{C}$ Morrissey, ${ }^{4} \mathrm{~F}$ Hakkak. ${ }^{1}$ The Royal Wolverhampton NHS Trust, Wolverhampton, UK; ${ }^{2}$ Graphnet Health Ltd, Milton Keynes, UK; ${ }^{3}$ Wolverhampton CCG, Wolverhampton, UK; ${ }^{4}$ Compton Hospice, Wolverhampton, UK

10.1136/thoraxjnl-2017-210983.166

COPD kills about 25000 people per year in England and Wales (DoH 2011). NICE guidelines (2010) suggest patients with end stage COPD should have access to palliative services. The national COPD audit showed on-site palliative care provision has increased from 50\% in 2008 to $87 \%$ in 2014 but less is known about out of hospital provision. The Gold Standard Framework (GSF) prognostic indicators assist clinicians to identify patients who are approaching end of life at an earlier stage enabling appropriate interventions to take place. These patients are more likely to receive well-coordinated and high quality care (GSF/RCGP 2011). The aim of our study was to map the number of QoF registered COPD patients, the frequency of COPD specific GSF indicators, the number included on primary palliative care registers ( $\geq 2$ GSF prognostic indicators) and the number reviewed by palliative care.

Methods A multidisciplinary group with membership from CCG, acute trust, hospice and the community team oversaw the project. After a data sharing agreement was completed, data was collated from across all health care sites in our area for COPD patients enabling us to confirm the number and type of GSF prognostic indicators for each patient and healthcare activity including palliative care reviews.

Results As of March 2016 there were 4999 COPD patients; $52 \%$ were male with an average age of 69.2 years. $25.7 \%$ of the patients $(n=1285)$ had $\geq 1$ GSF prognostic indicator. The most common indicator was MRC dyspnoea score of $4 / 5$ (65.5\%), followed by body mass index <20 (25.7\%). Of the 294 patients with $\geq 2$ GSF prognostic indicators $14.6 \%$ were on the GP palliative care register. $19.0 \%$ of the 294 patients had been reviewed by the palliative care team.

Conclusion GSF prognostic indicators in COPD are prevalent with breathlessness being the most common. Only a small proportion of appropriate patients were included on the palliative care register (14.6\%) with more being seen by palliative care teams than on the registers. Further work is needed to ensure effective communication and education is provided across the whole health care system to identify patients earlier who are approaching end of life.

\section{P25 LIVING WITH COPD: A PUBLIC AWARENESS AND SCREENING CAMPAIGN}

${ }^{1} \mathrm{MG}$ Crooks, ${ }^{2} \mathrm{~J}$ Thompson, ${ }^{3} \mathrm{~S}$ Platten, ${ }^{4} \mathrm{C}$ Evans, ${ }^{2} \mathrm{~S}$ Faruqi. ${ }^{1}$ Hull York Medical School, Hull, UK; ${ }^{2}$ Hull and East Yorkshire Hospitals NHS Trust, Hull, UK; ${ }^{3}$ City Health Care Partnership, Hull, UK; ${ }^{4}$ British Lung Foundation, London, UK

10.1136/thoraxjnl-2017-210983.167 